\title{
Dynamic behaviour of silks: Nature's precision nanocomposites
}

\author{
D.R. Drodge ${ }^{1}$, B. Mortimer ${ }^{2}$, C.R. Siviour ${ }^{1}$, and C. Holland ${ }^{2}$ \\ ${ }^{1}$ Department of Engineering Science, University of Oxford, Parks Road, Oxford OX1 3PJ, UK \\ ${ }^{2}$ Department of Zoology, University of Oxford, South Parks Road, Oxford OX1 3PS, UK
}

\begin{abstract}
Silk is often cited as a material worth imitating, due to its high strength and toughness. In order to produce a synthetic analogue, or enhanced natural version, the microstructural basis of these properties must be understood. Current understanding is that silk deforms through the detachment of nano-scale crystallites, in the manner of a damaged composite. This picture forms the basis for constitutive models, but validation data is limited to low strain-rates. Here we present a programme of research in which high-rate behaviour is studied through ballistic impact experiments. These have been applied to the silk of the Bombyx mori moth, as harvested from cocoons, and to the major ampullate thread of the golden orb weaver spider Nephila edulis. Longitudinal wave-speeds, and air drag coefficients, have been calculated for selected cases. Differences between the response of various silks and a similar synthetic fibre, nylon, are discussed, and future plans are presented.
\end{abstract}

\section{Introduction}

Spiders, silkworms and other arthropods produce silk, a protein fibre that possesses high toughness, strength and damage tolerance. A commonly stated truism is that spider dragline silk has a strength which exceeds that of steel, which is correct on a weight-for-weight basis. However it is the property of toughness that marks it out as superior to all other materials, including high-performance synthetic polymers such as Kevlar [1].

The mechanical properties of these natural fibres have been studied for many decades, predominantly at low strain-rates $[2,3]$. However, it is widely thought that silk production has been evolved to optimise energy storage and dissipation processes for prey capture [4], and furthermore to allow for environment-specific adaptation in the spinning process [5]. Energetic management processes i.e. storage and loss - are intrinsically time-dependent, so a study of the mechanical properties of silks at high strainrates is essential to complete the picture.

The superior toughness of silk is thought to be due to its hierarchical nanostructure [6]. The tertiary protein structure of the silk protein, fibroin in silkworms and spidroin in spiders, permits it to form nanoscale beadlike arrangements of folded beta-sheets [7]. These regions debond and then uncoil, dissipating energy in the process, giving rise to very high mechanical energy absorption, i.e. toughness. The nanoscale straining behaviour of silks is captured well using an activated debonding model [7]. Molecular dynamic simulations have been applied to the straining of silk proteins with some success [8]. However, in the absence of high strain-rate validation data, the validity of such models remains untestable.

Applying the Hopkinson Bar technique to silk fibres is difficult due to their slenderness, thus their low breaking force, which is of the order of $100 \mathrm{mN}$. Gauges capable of measuring such low forces are not installed in such apparatus as standard. Additionally, the Hopkinson bar requires a finite time for stress equilibrium to occur in the specimen [9], which is typically comparable to the time taken between zero stress and yield. Low-strain data, and time-dependent properties such as moduli, are therefore difficult to obtain.

However, being fibrous in form, silks are good candidates for a classical ballistic-impact experiment, devised by J. C. Smith and colleagues for use on textile yarns [10]. Transverse impact at moderate velocity produces both longitudinal and transverse waves in a fibre, the latter of which is easily recorded using a high-speed camera. Smith showed that with the application of a couple of assumptions, the transverse wave speed, along with the fibre tension and impact velocity, can be used to compute the longitudinal wave speed, which is a function of material properties rather than external constraints.

Through this approach, it is therefore possible to derive the Young's modulus for the material under impact rates. One can also observe the variation of longitudinal wave speed with applied tension, and thus determine the extent to which the mechanical properties of silks have been attuned to their typical service loads.

In the context of spider prey capture and web durability, the material properties of silk alone do not offer a full picture: rather, the action of air resistance in dissipating energy plays a significant role [11]. Transverse impact offers insight into this area as well, as the curvature of a deflected fibre can be used to estimate drag forces. For the range of available impact velocities, one can estimate a drag coefficient over a range of Reynolds numbers, and determine the degree to which spiders optimise their webs to take advantage of air drag in arresting prey impact whilst avoiding vulnerability to windy conditions.

\section{Materials}

Spider silk comes in a variety of forms, including acniform silk, used for egg protection; flagelliform silk used for the sticky capture spiral in webs; and ampullate or dragline silk, used for the structural parts of webs and for abseiling [12]. The ampullate silk, of which there are major and minor glands in most web spiders, is strongest, toughest 


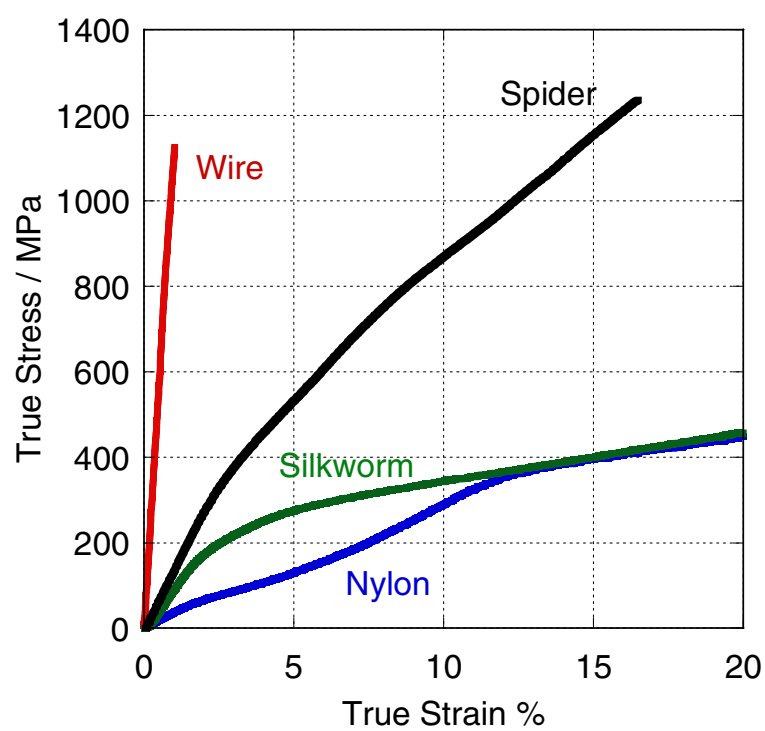

Fig. 1. Low rate $\left(\sim 10^{-2} \mathrm{~s}^{-1}\right)$ stress-strain properties of beryllium copper wire, $N$. edulis spider dragline, B. mori cocoon silk and medium tenacity nylon.

and most widely studied. Here we study the major ampullate silk from the golden orb weaver Nephila edulis, which is produced as a roughly circular fibre of spidroin protein of typical diameter 4-10 $\mu \mathrm{m}$ The Nephila spiders are studied due to their large size, and commensurately large and strong webs.

To obtain dragline silk, Nephila females were restrained and forcibly silked using a reeling device. Approximately 15-20 metres of silk was obtained per ampullate gland per spider per session. As the variation between individuals has not yet been fully characterised, only data from the silk from one individual spider is reported here.

The relatively short length of silk available from an individual spider places restrictions on the number of comparable experiments that can be performed. Silkworms, however, produce a fibre of similar underlying structure, if lower in performance, in much greater lengths. Bombyx mori silkworm fibre has been used for textile applications for centuries, having been bred specifically for this purpose. The worm produces a doublet fibre from two glands near its mouthparts, which has two tough fibroin protein cores, or "brins", and a weaker sericin skin. The fibre has a cross-sectional span of around 20 to $40 \mu \mathrm{m}$. In this study we used cocoon silk, unwound from a single silkworm cocoon, after treating with water to soften the interfibrillar cocoon bonds.

As Nephila and Bombyx silks are produced by organisms, and as such are subject to significant natural variation, a synthetic polymer - medium tenacity Nylon 6,6, supplied by Goodfellow Ltd - was also studied to provide a less variable, more geometrically consistent control specimen. To further validate the techniques, a metal wire - beryllium copper alloy - was also studied due to its low rate-dependence and high yield stress. Stressstrain curves and properties of these materials are shown in Fig. 1, with properties shown in Table 1.
Table 1. Properties of the four materials studied using transverse impact. Areas estimated from micrography, density from manufacturer specifications and literature, and Young;s modulus from Fig. 1.

\begin{tabular}{|c|c|c|c|}
\hline Material & $\mathbf{A} / \mu \mathbf{m}^{2}$ & $\varrho / \mathbf{k g ~ m}^{-3}$ & $\mathbf{E} / \mathbf{G P a}$ \\
\hline BeCu wire & 2060 & 8155 & 116 \\
\hline N. edulis & 14 & $1315[13]$ & 14.1 \\
\hline B. mori & 380 & $1315[13]$ & 10.6 \\
\hline Nylon & 490 & 1140 & 3.74 \\
\hline
\end{tabular}

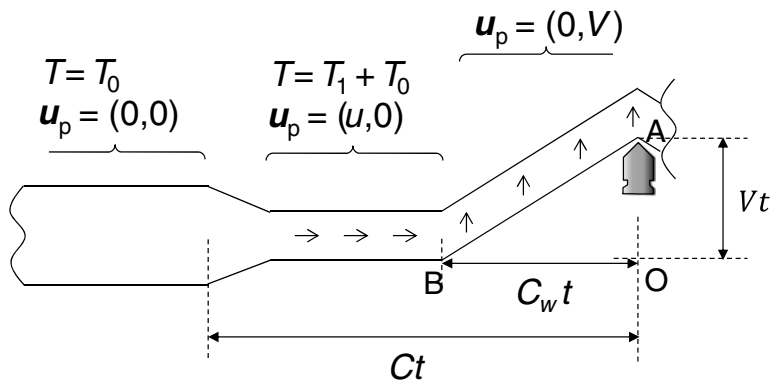

Fig. 2. Illustrating the motion of longitudinal wave at velocity $C$ and transverse wave at velocity $C_{w}$ - showing particle velocity $\mathbf{u}$ at each region of motion, and wavefront positions after time $t$.

\section{Methodology}

\subsection{Transverse impact}

The equations of motion for a notionally one-dimensional fibre allow for longitudinal and transverse wave propagation. When a rapid transverse displacement is applied to a point on a fibre, inertia will demand its axial elongation to ensure displacement compatibility. This elongation is propagated as a lontitudinal wave travelling at velocity $C$, and the lateral motion propagates as a transverse wave at velocity $C_{W}$. The situation is represented in Fig. 1 (after [14]). The fibre is initially under tension $T_{0}$, and the passage of the longitudinal wavefront increases the tension by $T_{1}$.

Smith's derivation, which is based on the triangle of displacements AOB shown in Fig. 2, leaves us with the relation shown in Eq. (1), derived by Smith [10]. The distances travelled by the projectile velocity, $V$, and measured transverse wave velocity, $C_{W}$, form the sides of the triangle, and the length of material that has flowed past the transverse wavefront forms the hypotenuse.

$$
V^{2}=\left(C_{w}+U\right)^{2}-C_{w}^{2}
$$

This is subject to few assumptions aside from that the strains are small enough for the usual wave-equations to hold. Solving for one of these three variables: impact speed, measured transverse wavespeed and longitudinal particle velocity; requires an appropriate hypothetical material model. For material characterisation, rather than model validation, such a model must be assumed.

So far two experimental schemes have been attempted: in one the fibre is held under low tension and the impact velocity is varied; in the other the velocity is held constant and differing tensions are applied. The former represents 


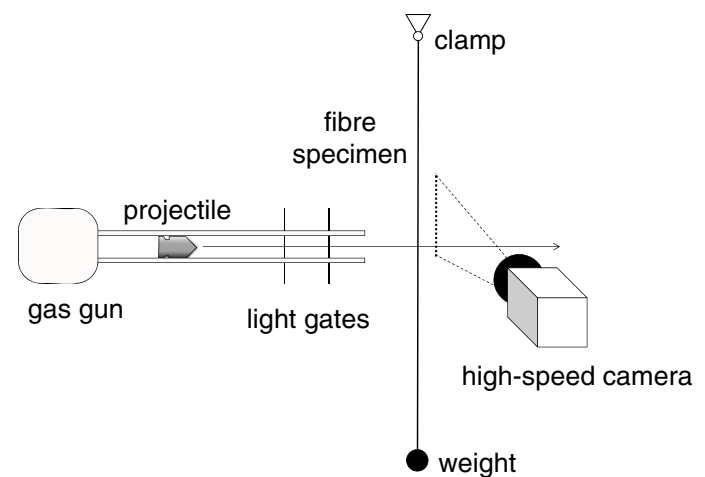

Fig. 3. Experimental setup for transverse fibre impact. Light gates trigger the camera and monitor projectile velocity.

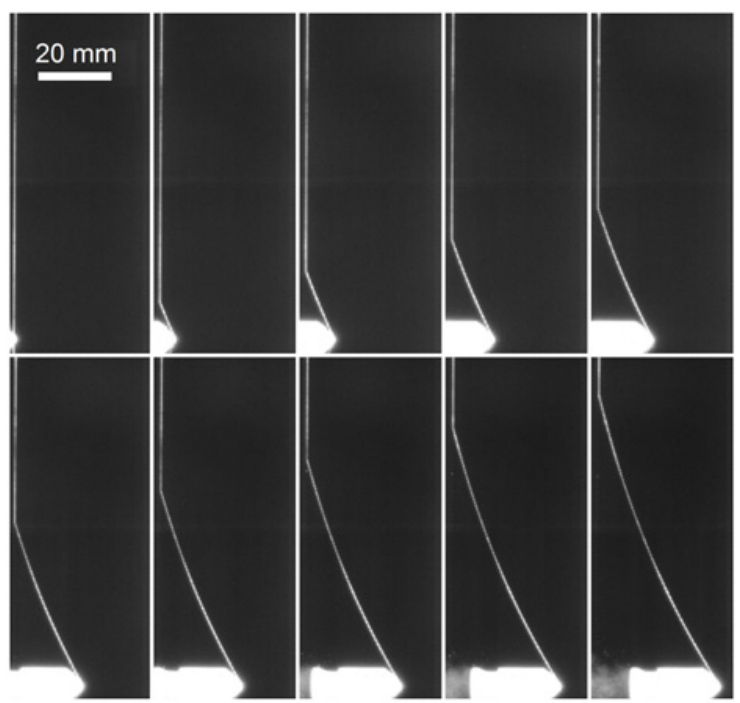

Fig. 4. High-speed photographs showing transverse wave motion in nylon fibre. Interframe time $21 \mu \mathrm{s}$.

a single step from near-zero strain to a post-impact state, and the latter represents a two-step loading path, with an initially equilibrated static load, followed by impact. In either case the experimental setup is the same: as shown in Fig. 3.

High speed imaging was performed using a Vision Research Phantom 7.1, and a Photron FASTCAM SA5, variously. An example frame-set of a struck fibre is shown in Fig. 4, from which impact velocity and transverse wave velocity can be measured, and the shape of the fibre after impact can be observed.

To determine the effect of fibre pre-stress on wave propagation, tension is varied by hanging various weights from the fibres prior to shooting. The resulting transverse wavespeeds are then interpreted under the assumption that the material can be treated as elastic during the impact. The equations that provide stress and strain due to elastic wave propagation are invoked $(\sigma=\varrho C U ; \varepsilon=U / C)$, and Eq. (1) becomes a quadratic in $C$ [15]. Here $A$ is the fibre cross-section, $T_{0}$ its static tension, and $\varrho$ its density.

$$
C^{2}+C\left(C_{w}^{2} / U+2 C_{w}-A T_{0} \varrho U\right)^{2}+A T_{0} \varrho=0
$$

The longitudinal wave speed can thus be obtained as a function of applied tension. The elastic impact step

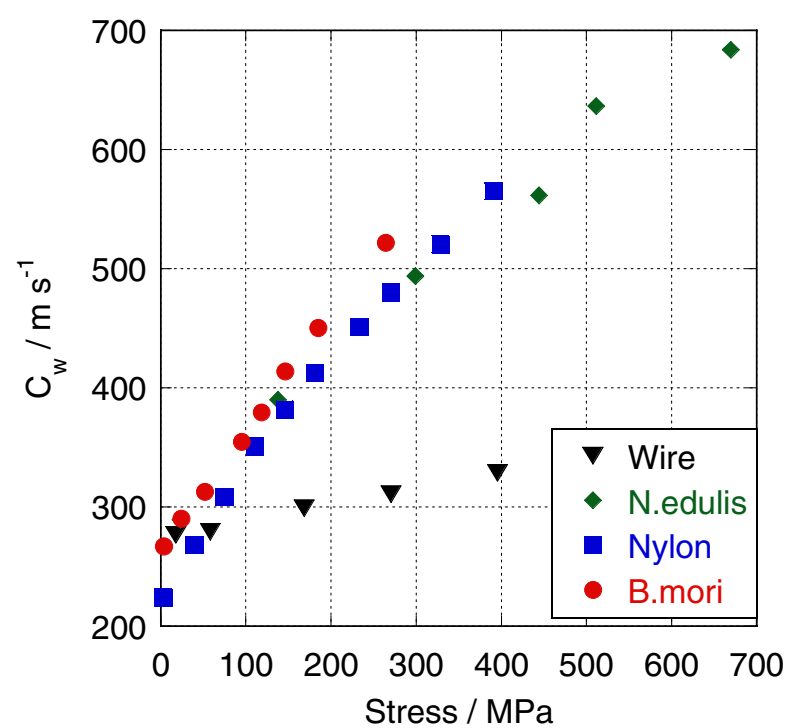

Fig. 5. Transverse wave-speed as a function of static stress $\left(T_{0} / A\right)$ for beryllium copper wire, cocoon silk (B. mori), spider dragline (N. edulis) and nylon. Impact speed is $120 \mathrm{~m} \mathrm{~s}^{-1}$ for all points.

assumption allows the additional stress and strain due to the impact of the projectile to be calculated, and a postimpact stress state to be plotted, forming a locus of postimpact states for the material [16].

Another analysis approach was devised by Smith, which allows a notionally "impact-rate" constitutive model to be derived from a series of impacts at different velocities [17]. Given a model $\sigma(\varepsilon)$, one can calculate the locus of possible $\left(V, C_{w}\right)$ co-ordinates for a given fibre tension using Eq. (1) and the von Karman equation for particle velocity [18].

As with Smith's original implementation, a tabulated stress-strain relation was posited, and iteratively modified until the predicted $\left(V, C_{w}\right)$ locus matched that obtained experimentally for each set of shots for a given material.

Transversely impacted fibres also provide an opportunity to observe the effect of high-velocity air drag on silk fibres. A simple analysis [19] provides a means of estimating the drag force on a fibre based on its curvature, from which a drag coefficient can be computed using Eq. (3).

$$
T / R=1 / 2 \varrho d C_{D} V^{2}
$$

Here, $R$ is the radius of curvature of the fibre at a given point, $T$ is its tension, $\varrho$ is the density of air, $C_{D}$ is the drag coefficient, $d$ is the fibre diameter, and $V$ is the component of fibre velocity that is normal to the fibre axis, at the point at which $R$ is measured.

\section{Results}

Transverse wave speed is plotted against fibre static stress and impact velocity for the various fibres in Fig. 5 and 6. The practical upper limit of static stress for each fibre was slightly above the yield stress, as the fibres tended to proceed to failure under such loads, prior to projectile impact.

Longitudinal wave speed is computed from Eq. (2), and plotted against varying static stress in Fig. 7. 


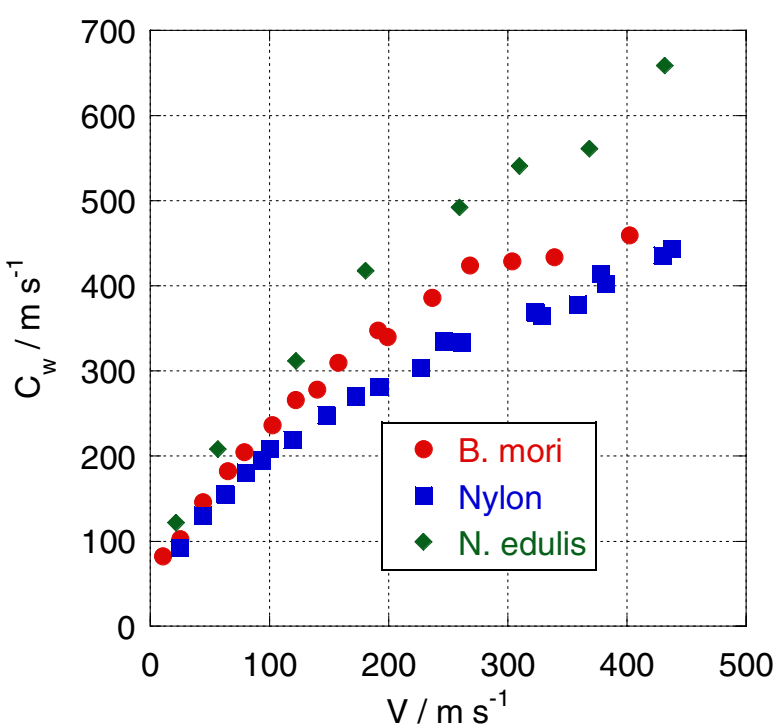

Fig. 6. Transverse wave-speed as a function of impact velocity for cocoon silk (B. mori), spider dragline (N. edulis) and nylon.

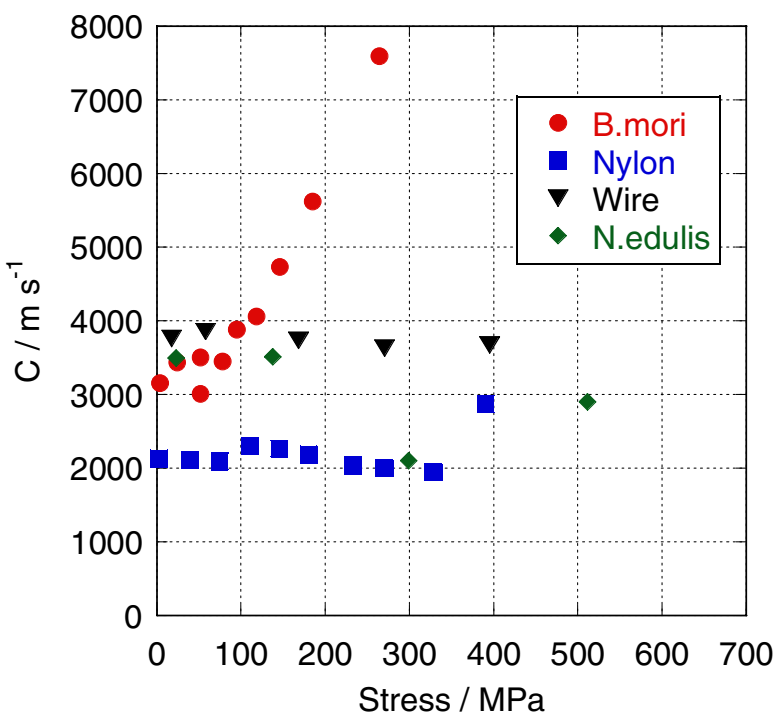

Fig. 7. Calculated longidutinal wave-speed as a function of static stress $\left(T_{0} / A\right)$ for beryllium copper wire, cocoon silk (B. mori), spider dragline (N. edulis) and nylon.

Stress-strain plots were produced for the materials on which the most data was available (cocoon silk and nylon) using the methods described above. These are shown separately for each material in Figs. 8 and 9. Where tension was varied, the path from the pre- to post-impact stress state is marked by an arrow. Where velocity was varied, and a model stress-strain curve assembled using Smith's approach, only the curve is shown.

The curvature of each material is illustrated in Fig. 10, showing stills of the fibre shape after the times shown. The tensions for these experiments are selected to produce broadly similar static stresses for each fibre.

Radii of curvature and local fibre velocities have been measured from the points marked, and the post-impact tensions calculated from the transverse wave velocity. Fibre diameter was estimated for each fibre individually.

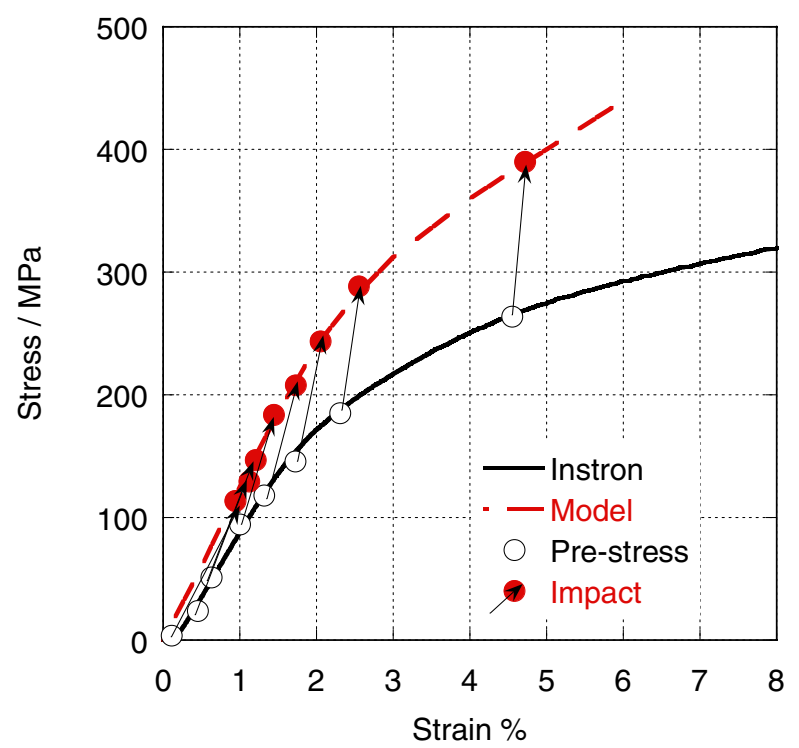

Fig. 8. B. mori cocoon silk stress-strain curves: "model" calculated from velocity method, "impact" states achieved after prestressing. Step from pre-stress to impact state shown by arrow.

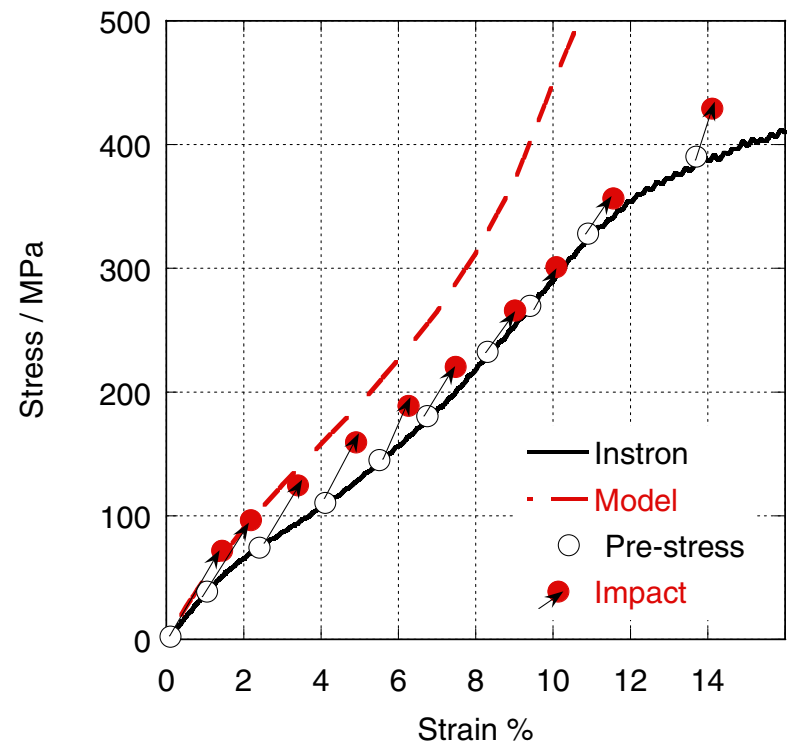

Fig. 9. Nylon stress-strain curves: "model" calculated from velocity method, "impact" states achieved after pre-stressing. Step from pre-stress to impact state shown by arrow.

Equation (3) was then used to compute a drag coefficient. The measurements and computed values, along with the Reynolds number (Re) and a literature value of $C_{D}$ taken from [20], are listed in table 2.

\section{Discussion}

The trends in transverse wave velocity seen in Fig. 5 confirm the trivial expectation that transverse waves travel faster in higher-tension fibres. Similarly, Fig. 6 illustrates that increasing velocities of impact lead to higher transverse wave velocities, this time because of the increasing impact stress, which also serves to increase the fibre tension. 


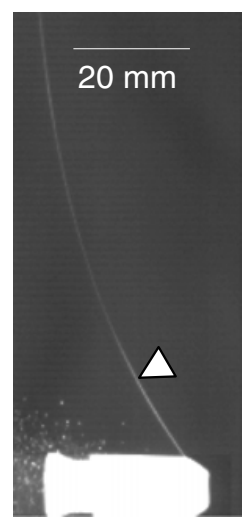

$\bar{N}$. edulis

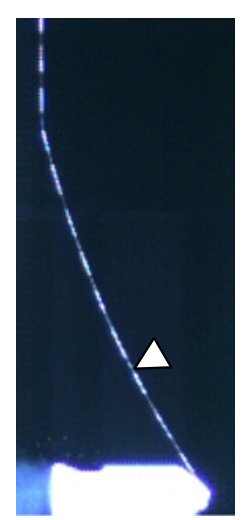

B. mori

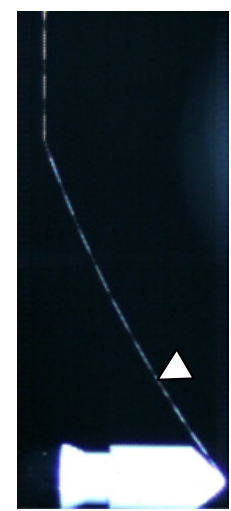

Nylon

Fig. 10. Framing images from $294 \mu$ s after projectile impact at $120 \mathrm{~m} \mathrm{~s}^{-1}$. Static tensions were $1.1 \mathrm{mN}$ for $B$. mori cocoon silk and nylon, and $1.7 \mathrm{mN}$ for $N$. edulis dragline silk. Triangles mark the points at which measurements of $V$ and $R$ were made.

Table 2. Measured local velocity and radius of curvature, with calculated post-impact tension $T$ and derived drag estimates, for the images shown in Fig. 10.

\begin{tabular}{|c|c|c|c|}
\hline & N.edulis & B. mori & Nylon \\
\hline $\boldsymbol{V} / \mathbf{m ~ s}^{-1}$ & 77 & 90 & 92 \\
\hline $\boldsymbol{R} / \mathbf{m}$ & 0.13 & 0.16 & 0.19 \\
\hline $\boldsymbol{T} / \mathbf{m N}$ & 3.1 & 24 & 34 \\
\hline $\boldsymbol{R} \boldsymbol{e}$ & 20 & 145 & 177 \\
\hline $\boldsymbol{C}_{\boldsymbol{D}}$ & 2.1 & 1.5 & 1.4 \\
\hline $\boldsymbol{C}_{\boldsymbol{D}}[20]$ & 2.0 & 1.4 & 1.4 \\
\hline
\end{tabular}

Longitudinal velocity for each material (Fig. 7) shows differing dependences on static stress. The metal shows a wave speed of around $4000 \mathrm{~ms}^{-1}$ which does not increase with increasing pre-stress. The wave-speeds of nylon and cocoon silk begin to increase when the pre-stress exceeds the low-rate yield stress (see Fig. 1), indicating some underlying structural change as the fibres yield.

The present lack of comparable spider gland silk, thus small amount of data, makes it difficult to comment on such trends in spider silk. However the wave-speed obtained at the lowest pre-stress is useful as it can be used to compare impact-rate tensile modulus (calculated to be $16 \mathrm{GPa}$ ) to the low-rate value of $14 \mathrm{GPa}$ (see table 1). For cocoon silk the impact-rate modulus is approximately $15 \mathrm{GPa}$, which presents a slightly greater increase from the low-rate value of $10 \mathrm{GPa}$. For nylon the impact-rate modulus is approximately $6 \mathrm{GPa}$, which is a large increase from the low-rate value of $3.4 \mathrm{GPa}$. The metal wire shows no significant difference between low-rate and impact-rate moduli.

The computed stress-strain curves shown in Figs. 8 and 9 demonstrate the differences between the velocityand tension- variants of the transverse impact experiment. The velocity-derived model shows a higher stress-strain relationship than the low-rate curve. The stress-states achieved after impact in the tension-varying experiments are remarkable in cocoon silk as they are coincident with the velocity-derived model, whereas in nylon they are not. It remains to be seen, subject to additional experiments,

whether spider silk behaves more like the former or the latter.

The calculated drag coefficients shown in table 2 compare very well with the values stated by Sheard et al for airflow around an infinite cylinder [20], validating the use of their data in modelling web behaviour. Those authors indicate a transition from steady to unsteady flow occurs when $\operatorname{Re} \sim 50$, which places Nephila silk in the steady flow regime for these experiments, which arguably represent an extreme airflow for a spider web. At the sort of velocities seen during prey capture, of the order of $10 \mathrm{~ms}^{-1}$, the Reynolds number will certainly lie within the stable regime. Further measurement of the images obtained during these experiments is expected to provide additional information on the role of air drag in the energy dissipation process in spider webs, and how these operate in tandem with the material dissipative processes.

\section{Conclusions}

The transverse impact technique allows for the measurement of fibrous material response under impact-rate conditions, and is well suited to studying silk fibres. Longitudinal wave speed, thus tensile modulus, was found to increase with increasing pre-stress in silk and nylon, whereas purely elastic materials would be expected to show no such trend. The initial moduli of these polymers were also seen to be higher than those obtained using low-rate mechanical testing apparatus, although the proportional increase was less in the silks.

The technique can also be used to produce a stressstrain curve from a series of experiments, through two different loading path strategies. The agreement seen in silkworm silk, and difference seen in nylon, indicates a fundamental difference in path sensitivity between these materials.

Air drag was computed from high-speed images, and confirms the validity of a Reynolds-number based prediction of drag coefficient even at such low fibre diameters and high velocities. Further study will explore the influence of turbulence at higher Reynolds numbers, achieved by increasing the impact velocity.

\section{Acknowledgements}

This research is supported by The Leverhulme Trust (F/08705/D), the US Air Force Office of Scientific Research (F49620-03-10111), the European Research Council (SP2-GA-2008-233409) and Magdalen College. Prof. David Porter and Prof. Fritz Vollrath are thanked for insightful comments and suggestions. The authors are grateful to Richard Duffin and the workshop at the Department of Engineering Science for their invaluable contributions to the design and execution of experiments.

\section{References}

1. A. Sponner, Entomol. Res. 37, 238 (2007)

2. J. Gosline, P. Guerette, C. Ortlepp, K. Savage, J. Exp. Biol. 2023295 (1999) 
3. Y. Yang, X. Chen, Z. Shao, P. Zhou, D. Porter, D. P. Knight, F. Vollrath, Adv Matls 1784 (2005)

4. C. Craig, Annu. Rev. Entomol. 42231 (1997)

5. F. Vollrath, Int. J. Bio. Macromol. 2481 (1999)

6. C. Holland, F. Vollrath, A. Ryan, O. Mykhaylyk, Adv. Mater. 24105 (2012)

7. D. Porter, F. Vollrath, Adv. Mater. 21487 (2009)

8. A. Nova, S. Keten, N. Pugno, A. Redaelli, M. Buehler, Nano Lett. 102626 (2010)

9. G. Gray, in ASM Handbook vol 8, p. 463 (ASM International, 2000)

10. J. Smith, F. McCrackin, H, Schiefer, Text. Res. J. 26 821 (1958)

11. L. Lin, D. Edmonds, F. Vollrath, Nature 373146 (1995)

12. R. Foelix, Biology of Spiders (Oxford University Press, 2011)
13. J. Zemlin, "A study of the mechanical behavior of silks", (US Army Natick Laboratories, MA, 1968)

14. D. Roylance, A. Wilde, G. Tocci, Text. Res. J. 4334 (1973)

15. D. Prevorsek, H. Chin, D. Kwon, J. Field, J. Appl. Polym. Symp. 4744 (1991)

16. D. Drodge, B. Mortimer, C. Holland, C. Siviour, J. Mech. Phys. Solids (in review)

17. J. Smith, C. Fenstermaker, P. Shouse, Text. Res. J. 31 $721(1961)$

18. T. von Karmen, P. Duwez, J. Appl. Phys 21987 (1950)

19. J. Smith, C. Fenstermaker, P. Shouse, J. Res. Nat. Bur. Standards C 68177 (1964)

20. G. Sheard, K. Hourigan, M. Thompson, J. Fluid Mech. 526257 (2005) 